\title{
MultiGranular: An effective Service Composition Infrastructure for Multi-tenant Service Composition
}

\author{
Huihui Cai and Lizhen Cui ${ }^{*}$ \\ School of Computer Science and Technology Shandong University, \\ Jinan, 250101, China
}

chh_121@126.com,clz@sdu.edu.cn

\begin{abstract}
As a common delivery model in cloud computing, Sans applieations are becoming increasingly popular. Multi-tenancy is a key charactenistics of S\&aS applications. Service composition plays a main role in SaaS applications becquse of frequent composability and reusability of software services. With the development of cloud computing, one side, there are an increasing number of available services, other side, tenant individual and diverse requirement become more intense, which makes service gomposition tend to be more complex. This paper proposes an effective infrastructure for service composition, MultiGranular, which provides a semantic basis for Gulti-tenant service composition. The MultiGranular supports the characternstic of hierarchy, uncertain correlation, inheritance and versioning, effectively responding to the requirements of complexity of service composition for cloud computing Sala applicatigns. The computing process of MultiGranular includes three steps: basic service clustering, correlation mining and hierarchy building. MultiGranular makes it possible to chinge disorder services into the hierarchy and ordered clustering services, and makes it feasy to develop multi-tenant SaaS applications. The experimental results demonstrate that by utilizing the proposed method, complexity of multi-tenant service composition are solved and the efficiency of service composition has been improved greatly.
\end{abstract}

Keywords: cloud computing, multi-tenant, service composition, hierarchy, uncertain correlation, inheritance and versioning

\section{Introduction}

As the de elopment of cloud computing and service computing, SaaS (Software as a Serrice) is widely used for bringing benefits to both software service providers and tenants. Service composition has become the dominant paradigm that utilizes services as fundamental elements for developing SaaS applications efficiently. However, one side, there is an increasing number of available services in large enterprise or domain, other side, tenant's individual and diverse requirement become more intense, which makes service composition tend to be more complex. In cloud computing, the complexity of multi-tenant service composition reflects in the following four aspects:

Multi-granularity. Business requirements are variant, and some are abstract and some are specific. Requirements show characteristic of multi-granularity. For example, someone submits his requirement as "travel plan" while others may say they want "book flight". How to satisfy different users' multi-granularity requirements is always an urgently solved problem. 
Complexity of correlation. In the cloud SaaS model, services are sharing and isolation. Correlation relationships between services are more complex. In addition, the uncertainty of correlation relationships between services tends to be significantly increased.

Massive individuation. In the cloud environment, almost every tenant has special requirements for services in terms of UI, business process, data and so on. It indicates characteristic of individuation which varies a lot.

Frequency of evolution. Services always frequently evolve from one version to another version due to the change in business requirements. So it is necessary to effectively manage these services before and after evolution.

\section{Related work}

Over the last several years, different composition strategies have been proposed. [1] proposed an automated service composition approach based on service community. Task templates and service pools are included in the community to facilitate service discovery and composition. However, it is not poss ble to predefine all the templates. [2] proposed an uncertain QoS-aware skyline service selection-method based on cloud model. With this method, the reliability and rear-time performance of service selection have been guaranteed. [3] proposed a nêtwork-aware serviee composition method which considers the QoS of the network except the QoSof the service itself. It efficiently computes compositions with much 10wer latency. 14] built an extended quick service query list using WordNet and mulliple heterogeneous ontologies to record service information and their associated semantie concepts towards more realistic service composition. The method alleviates users or developers from the burden of using complicated semantic service composition and can select and compose services to construct plans automatically. [5] proposed a multiobjective optimization framework for SLA-aware service composition leveraging a multiobjective genetic algorithm. [6] proposed an extended graph-planning based Top-K service composition method. By using service indexes and auxiliary nodes in extended planning graph, Top-K composition service cap be found through one search.[7] put forward a matching algorithm SMA (Seryice Matching Algorithm) between cloud computing services of multiple input/output parameters, which considers the semantic similarity of concepts in parameters based on WordNet. [8] proposed a framework of service composition in multi-cloud base environments and three different cloud combination methods considering finding feasible composition sequence that contains minimum clouds. [9] proposeda QoS-based selection of multi-granularity web services for the composition, which allows services of multi-granularity available for selection. [10] proposed a service composition optimizing method which was user-oriented and based on the QoS value. Based on the fact that users usually put forth a coarse-granularity request. [11] proposed an on-demand service organization and recommendation method based on role, goal, process and service model for a domain problem to satisfy user's personalized and diverse requirements. [12] proposed an event-based web service composition in order to satisfy user's multiple requirements and be realized easily.

Though these methods above can achieve good results to some extent, they have not taken multi-tenant into consideration. Multi-tenancy is a key characteristics of SaaS applications, and there are some issues that traditional service composition approaches have not yet well addressed like multi-granularity requirements, complexity of correlation, massive individuation and frequency of evolution. With the lessons and 
experiences from the traditional service composition approaches, we present an effective infrastructure for multi-tenant service composition.

\section{Overview}

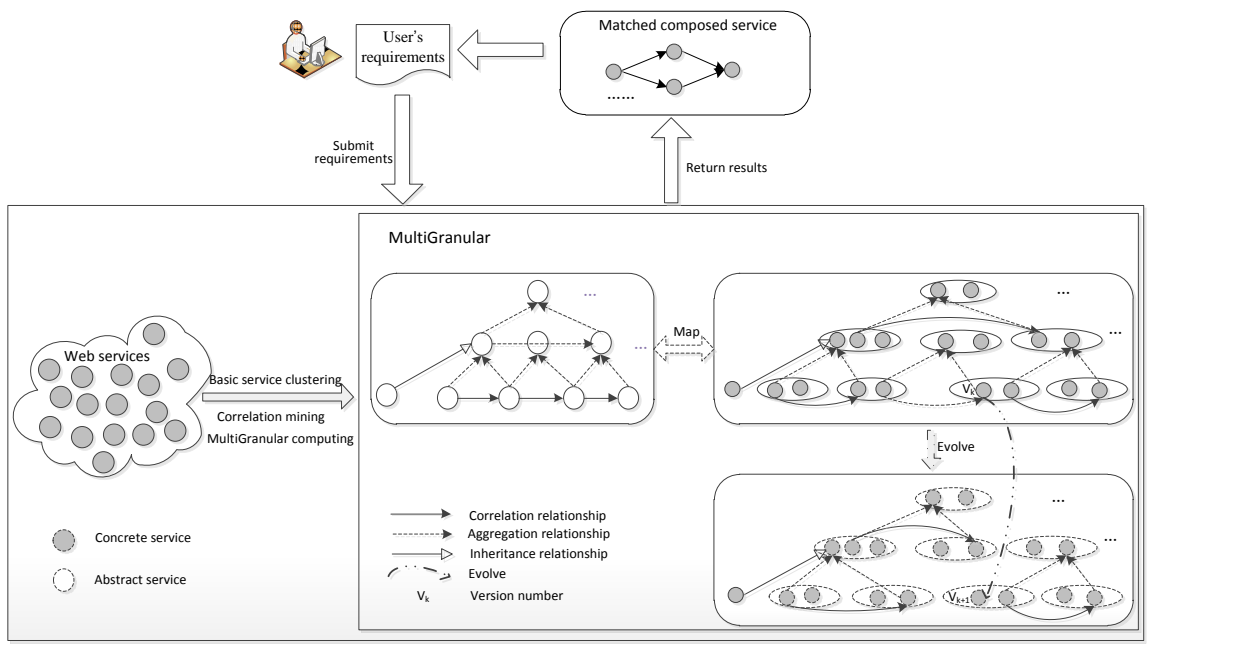

Figure 1. The Architecture ofMultiGranular

The architecture of MultiGranular is shown in Figure 1. In order to respond to the complexity of multi-tenant service composition in cloud computing, we give the corresponding solving strategjes:

Hierarchy. Considering from similar, correlation and aggregation relationships of the granularity among services, the process of MultiGranular computing is introduced, which includes basio service clustering, correlation mining and hierarchy building, which makes volymes of disorder services into the hierarchy and ordered clustering services, effectively responding to multi-granularity service composition requirements.

Uncertain correlation Uncertain of correlation relationships are measured from the aspect of correlation degree between services. The computing of correlation degree is based on matching between concepts of services message and similarity measurement.

Inheritance. Tenants can firstly build sub service reflecting their individual requirement by inheriting on the mostly similar service with their requirements with least inheritance operation. Then corresponding concrete service will be got from which the inherited service maps tofor customization. Inheritance is realized by modifying attributes of services, such as UI, business process, descriptive definition and data schema to satisfy tenants' individual requirements. In section 5 we will introduce some inheritance operations.

Versioning. The frequent evolution of services to meet business requirements changed always leads to many versions of the same original service. Therefore, it is necessary to realize version management mechanism. 


\section{MultiGranular Computing}

As Figure 1 shows, MultiGranular includes two hierarchical models, in which abstract services maps to concrete services mutually. Formally, it is represented as a quintuple, $\left(S, R_{s}, R_{c}, R_{a}, R_{i}, D e\right)$, where:

Definition 1. Service $S^{(v)}=$ (UI, Business process, descriptive definition, data)

UI provides the uniform entrance for accessing service and describes the functional module and layout. Business process is the specific process for realizing particular goal and includes atomic process and composite process. It is consisted of services and flow relationships between them. Descriptive definition mainly declares the functionalities, such as input and output message and their data types. Data schema is consisted of a set of data items. $v$ is the version number.

Definition 2. Similar relationship $R_{s}$. Services that can realize similar functionality because of similar semantic concepts are similar. Similar relationship between servides is defined as

$$
S_{i} \approx S_{j}, \text { Similar }\left(S_{i}, S,\right) \geq 0
$$

There are many methods to compute similarity degree between services. In this paper, we compute the similarity degree based on their distance in the ontology.

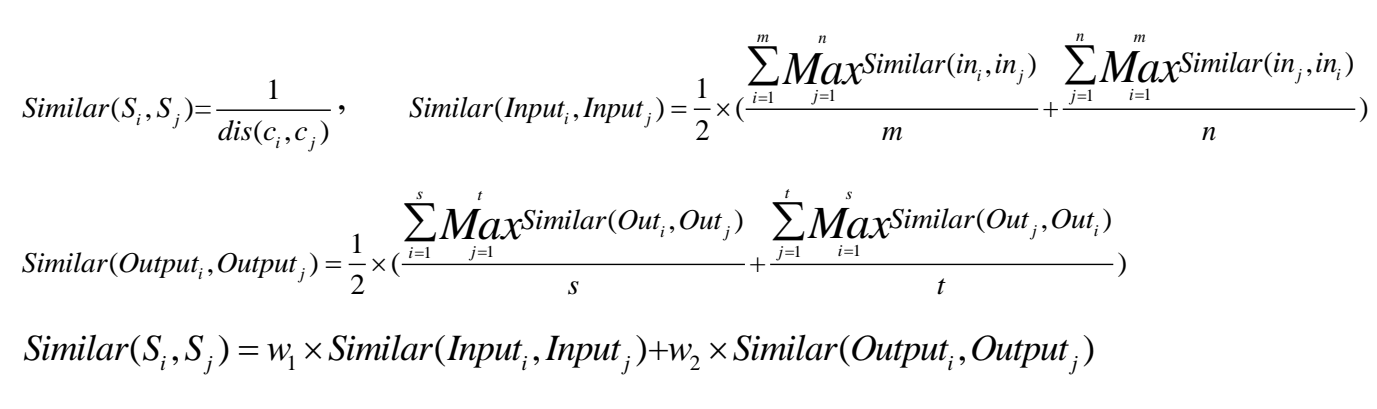

In the MultiGranular, we firsty divide service set into several groups, services in each of which haye relatively high similarity in functionality while services between different of which vary a lot. After basic services clustering, each service cluster can be represented as an abstractservice through extracting the same concept respectively in the input and outputmessage of services in the same cluster.

Definition 3. Correlation relationship $\mathrm{R}_{\mathrm{c}}$. Correlation relationship is the basis of service composition. According to the matching relationship between concepts of input and output message in the ontology, two forms of correlation relationships between services are defined as follows:

$$
\begin{aligned}
& \text { (1) } S_{i} \stackrel{R_{c}}{\longrightarrow} S_{j} \text {, if } S_{i} \text {.Output } \supseteq S_{j} \text {.Input, Similar }\left(S_{i} \text {.Output, } S_{j} . \text { Input }\right) \geq \delta \\
& \text { (2) } S_{1} \cup S_{2} \cup \cdots \cup S_{n} \stackrel{R_{c}}{\longrightarrow} S_{j} \text {, if } \begin{array}{l}
S_{1} \text {.Output } \cup S_{2} . \text { Output } \cup \cdots \cup S_{n} \text {.Output } \supseteq S_{j} \text {.Input, } \\
\forall S_{i}, C D\left(S_{i}, S_{j}\right)=\operatorname{Similar}\left(S_{i} \text {.Output, } S_{j} \text {.Input }\right) \geq \delta
\end{array}
\end{aligned}
$$

In the second step of building MultiGranular, the two forms of correlation relationship will be mined among abstract services according to semantic matching.

Definition 4. Aggregation relationship $\mathrm{R}_{\mathrm{a}}$. As coarse-grained services can hierarchically decompose to fine-grained services that have correlation relationship, we measure the two forms of aggregation relationship in the aspect that one service can realize composition function of services correlated. 


$$
\begin{aligned}
S_{i}, S_{j} \stackrel{R_{a}}{\longrightarrow} S_{k} \text {,if } \quad & S_{i} \stackrel{R_{c}}{\longrightarrow} S_{j} \\
& \left.S_{i} \cdot \text { Input } \approx S_{k} \text { Input namely } \forall . S_{i} \text { Similar } S_{i} \text { (nput } S_{k} \text { Input. } \geq \delta\right) \\
& S_{i} \cdot \text { Output } \approx S_{k} \text { Output namely } \forall \cdot S_{i} \text { Similar } S_{i} \text { Output } S_{k} \text { Qutput } \geq \delta
\end{aligned}
$$

$$
S_{1}, S_{2}, \cdots, S_{n}, S_{j} \stackrel{R_{c}}{\longrightarrow} S_{k} \text {, if }
$$

$S_{1} \cup S_{2} \cup \cdots \cup S_{n} \stackrel{R_{c}}{\longrightarrow} S_{j}$

$S_{1}$.Input $\cup S_{2}$.Input $\cup \cdots \cup S_{n}$.Input $\approx S_{k}$.Input, namely $: \forall S_{i}$, Similar $\left(S_{1}\right.$.Input $\cup S_{2}$.Input $\cup \cdots \cup S_{n}$.Input, $S_{k}$.Input $) \geq \delta$

$S_{j}$.Output $\approx S_{k}$.Output, namely $: \forall S_{i}$, Similar $\left(S_{j}\right.$.Output,$S_{k}$.Output $) \geq \delta$

After the first two steps in MultiGranular building, correlation relationships among abstract services are mined. For correlated services, check whether there are coarse-grained services that can complete the composition functionality of them. Once found, aggregation relationships among them are built hierarchically. As each abstract serviee is mapped to the concrete service cluster, hierarchy building of service clusters /will be correspondingly finished.

Definition 5. Inheritance relationship $\mathrm{R}_{\mathrm{i}}$. Services that have general attributes are called parent services; services that reflect individual requirement through inherited operations without changing logic realization are called child services.

Definition 6. De: $S \rightarrow G$ representing adecomposifion of service $\mathrm{s} \in \mathrm{S}$ to a set of dags G. De $(s)=(V, E) V=S, E \subset\left\{R_{c}, R_{a}(\}, \forall s_{i}, s_{j} \in S,\left(s, s_{i}\right) \in R_{a},\left(s_{i}, s_{j}\right) \in R_{c}\right.$.

\section{Implementation of Individuation and Evolution}

\subsection{Inheritance}

Inheritance can take place in several aspects of service, such as UI, business process, descriptive definition and dataschema. Below is the corresponding inheritance operation for each attribute of service.

(1)For UI inheritance, there are two kinds of inheritance operation:

Change contents: For example, add new module or adjust font size.

Change structure For example, adjust the layout of some modules or change the position of some images and menus.

(2)For business process inheritance, there are six kinds of inheritance operation:

dd business process: assume the original business process is $\mathrm{BP}_{1}=\left(\mathrm{i}_{1}, \mathrm{~S}_{1}, \mathrm{o}_{1}, \mathrm{~F}_{1}\right)$ and $\mathrm{BP}_{2}=\left(\mathrm{i}_{2}, \mathrm{~S}_{2}, \mathrm{O}_{2}, \mathrm{~F}_{2}\right)$ is the business process to be added to. Each business is represented as a quadruple: input, service set, output, and data flow relationship. Assume i and o are separately start service and end service in the business process. There are three kinds of inheritance operation:

Sequence adding: We call $\mathrm{BP}=(\mathrm{i}, \mathrm{S}, \mathrm{o}, \mathrm{F})$ as the business process got through sequence adding $\mathrm{BP}_{2}$ to $\mathrm{BP}_{1}$, in which, $\mathrm{S}=\left\{\mathrm{S}_{1} \backslash \mathrm{o}_{1}\right\} \cup \mathrm{S}_{2}, \mathrm{~F}=\left\{\left(\mathrm{s}_{\mathrm{i}}, \mathrm{s}_{\mathrm{j}}\right) \in \mathrm{F} 1 \mid \mathrm{s}_{\mathrm{j}} \neq \mathrm{o}_{1}\right\} \cup\left\{\left(\mathrm{s}_{\mathrm{i}}, \mathrm{i}_{2}\right) \mid\left(\mathrm{s}_{\mathrm{i}}, \mathrm{o}_{1}\right) \in \mathrm{F}_{1}\right\} \cup \mathrm{F}_{2}$.

Parallel adding: We call $\mathrm{BP}=(\mathrm{i}, \mathrm{S}, \mathrm{o}, \mathrm{F})$ as the business process got through parallel adding $\mathrm{BP}_{2}$ to $\mathrm{BP}_{1}$, in which $\mathrm{S}=\mathrm{S}_{1} \cup \mathrm{S}_{2} \cup\{\mathrm{i}, \mathrm{o}\}, \mathrm{F}=\mathrm{F}_{1} \cup \mathrm{F}_{2} \cup\left\{\left(\mathrm{i}, \mathrm{i}_{1}\right),\left(\mathrm{i}, \mathrm{i}_{2}\right),\left(\mathrm{o}_{1}, \mathrm{o}\right),\left(\mathrm{o}_{2}, \mathrm{o}\right)\right\}$. 
Choice adding: We call $\mathrm{BP}=(\mathrm{i}, \mathrm{S}, \mathrm{o}, \mathrm{F})$ as the business process got through choice adding $\mathrm{BP}_{2}$ to $\mathrm{BP}_{1}$, in which $\mathrm{S}=\mathrm{S}_{1} \cup\left\{\mathrm{S}_{2} \backslash\left\{\mathrm{i}_{2}, \mathrm{O}_{2}\right\}\right\}, \mathrm{F}=\mathrm{F}_{1} \cup\left\{\left(\mathrm{i}_{1}, \mathrm{~s}_{\mathrm{i}}\right) \mid\left(\mathrm{i}_{2}, \mathrm{~s}_{\mathrm{i}}\right) \in \mathrm{F}_{2}\right\} \cup\left\{\left(\mathrm{s}_{\mathrm{i}}, \mathrm{s}_{\mathrm{j}}\right) \in \mathrm{F}_{2} \wedge \mathrm{s}_{\mathrm{i}} \neq \mathrm{i}_{2} \wedge \mathrm{s}_{\mathrm{j}}\right.$ $\left.\neq \mathrm{o}_{2}\right\} \cup\left\{\left(\mathrm{s}_{\mathrm{i}}, \mathrm{O}_{1}\right) \mid\left(\mathrm{s}_{\mathrm{i}}, \mathrm{o}_{2}\right) \in \mathrm{F}_{2}\right\}$.

Delete business process: assume the original business process is $\mathrm{BP}=(\mathrm{i}, \mathrm{S}, \mathrm{o}, \mathrm{F}), \mathrm{BP}_{1}$ is the business process to be deleted; $\mathrm{BP}_{2}$ is the business process that has parallel or choice relationship with $\mathrm{BP}_{1}$ in $\mathrm{BP}$. There are three kinds of inheritance operation:

Sequence deleting: We call $\mathrm{BP}^{\prime}=\left(\mathrm{i}, \mathrm{S}^{\prime}, \mathrm{o}^{\prime}, \mathrm{F}^{\prime}\right)$ is the business process got through sequence deleting $\mathrm{BP}_{1}$ from $\mathrm{BP}$, in which, $\mathrm{S}^{\prime}=\mathrm{S} \backslash\left\{\mathrm{S}_{1}\right\}, \mathrm{i}^{\prime}=\mathrm{i}, \mathrm{o}^{\prime}=\mathrm{i}_{1}, \mathrm{~F}^{\prime}=\mathrm{F} \backslash\left\{\left(\mathrm{s}_{\mathrm{i}}, \mathrm{i}_{1}\right)\right\} \backslash \mathrm{F}_{1}$.

Parallel deleting: We call $\mathrm{BP}^{\prime}=\left(\mathrm{i}^{\prime}, \mathrm{S}^{\prime}, \mathrm{o}^{\prime}, \mathrm{F}^{\prime}\right)$ is the business process got through patallel deleting $\mathrm{BP}_{1}$ from $\mathrm{BP}$, in which, $\mathrm{S}^{\prime}=\mathrm{S} \backslash\left\{\mathrm{S}_{1}\right\}, \mathrm{i}^{\prime}=\mathrm{i}, \mathrm{o}^{\prime}=\mathrm{o}_{2}, \mathrm{~F}^{\prime}=\mathrm{F} \backslash\left\{\left(\mathrm{i}, \mathrm{i}_{1}\right),\left(\mathrm{o}_{1}, \mathrm{o}\right)\right\} \backslash \mathrm{F}_{1}$.

Choice deleting: We call $\mathrm{BP}^{\prime}=\left(\mathrm{i}^{\prime}, \mathrm{S}^{\prime}, \mathrm{o}^{\prime}, \mathrm{F}^{\prime}\right)$ is the business process g6t though choice

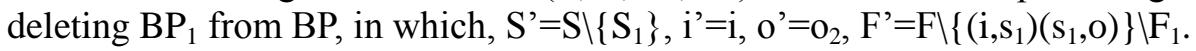

(4)For descriptive definition inheritance, there are two Rinds of inheritance operation:

Add parameter $i_{j}$ or $o_{j}: \mathrm{I}=\mathrm{I} \cup\left\{\mathrm{i}_{\mathrm{j}}\right\}$ or $\mathrm{O}=\mathrm{O} \cup\left\{\mathrm{o}_{\mathrm{j}}\right\}$ given the condition the added parameter does not affect the functionality.

Delete $\mathrm{i}_{\mathrm{j}}$ or $\mathrm{o}_{\mathrm{j}}$ : $\mathrm{I}=\mathrm{I} \backslash\left\{\mathrm{i}_{\mathrm{j}}\right\}$ or $\mathrm{O}=\mathrm{O} \backslash\left\{\mathrm{o}_{\mathrm{j}}\right\}$ giren the conditionthe added parameter does not affect the functionality.

(5) For data schema inheritance, there are threekinds of inheritance operation:

Add data item $d_{i}$ : DS=DS $\cup\left\{d_{i}\right\}, \mathrm{DS}=$ (name, birthdate). For example, add a data item named affiliation in a registry service table schema.

Delete data item $d_{i}$ : DS $=\mathrm{DS}\left\{\left\{d_{i}\right\}\right.$, delete the attribute description of $d_{i}$ and the dependency between data items.

Change data item $a_{i}$; For example, you can rename or change the type of the data item.

\subsection{Versioning}

Service versioning is used to identify different versions of the same service. The way how a version is determined and which characteristics included in the service of the version is defined by the service developer. Particularly, attributes about the version such as the number, difference from the service of original version and the description must be recorded so that users are provided many choices when selecting versions. Once the logic realization of the service ischanged, a new version of the same service is generated. By applying versioning technique to services, different users can have different versions of the same service, in which some are the latest available version of the service while a limited number of users lag one or more versions behind. Once the new version of the service is released, some users may migrate to the new version while some users may stay on the original version for incompatibility.

\section{Experimentation}

The approach proposed in the paper is applied to the social security service system development based on SaaS. Developers develop services according to the service specification and publish them in the system. Those massive disorderly services are then organized by the approach as an orderly structure. When developers or tenants need to develop new social security application, it can be achieved quickly through service 
composition using the approach. The experiment system contains thousands of services and runs on a computer running win 7 with a $2.33 \mathrm{GHz}$ Dual Core processor and $2 \mathrm{~GB}$ memory. From the point of view of functions needed to achieve, this system can be divided into two main modules: (1) the building of MultiGranular; (2) the service composition system. As the first module has been previously shown, we focus on introducing the service composition system here.

\subsection{Service Composition System}

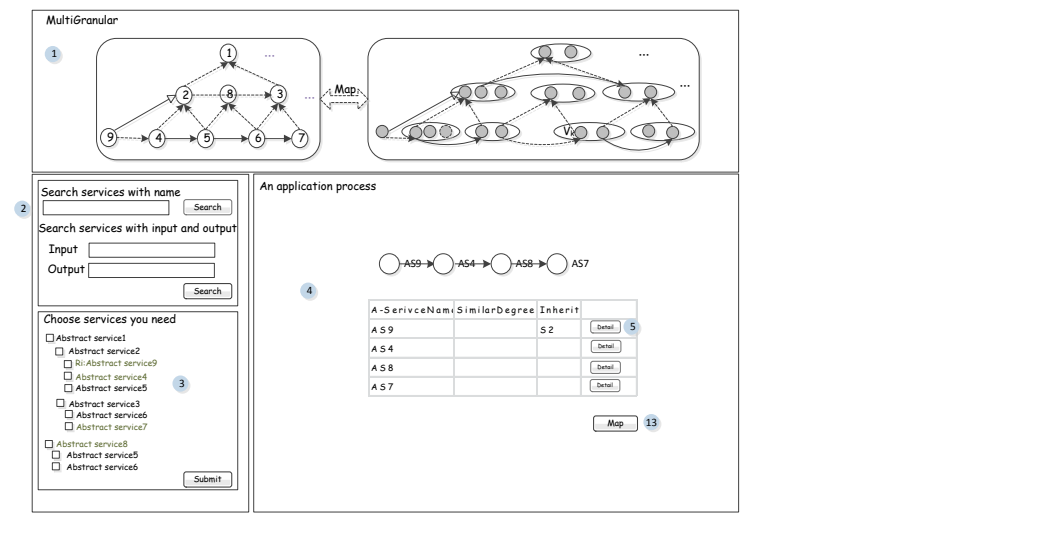

Figure 2. Service, Composition Environment

As shown in Figure 2, when tenahts login in to the system, MultiGranular will be displayed to them in graphical form (1) . In the lower left of the interface, there are different categories of services, which coyer the business functronality scope. Tenants can select services they need through clicking checkboxes aside by the abstract services or get services they need by clicking the search button after inputting the input parameters(2) of services they need as shown in the green sepvice(3). Onde the Submit button is clicked, service composition plans will be shown in the right area. Each abstract service in the plan is represented as a table, and each row is associated to member service (4). The specified information of concrete services it maps can bechecked by clicking Detail button (5).

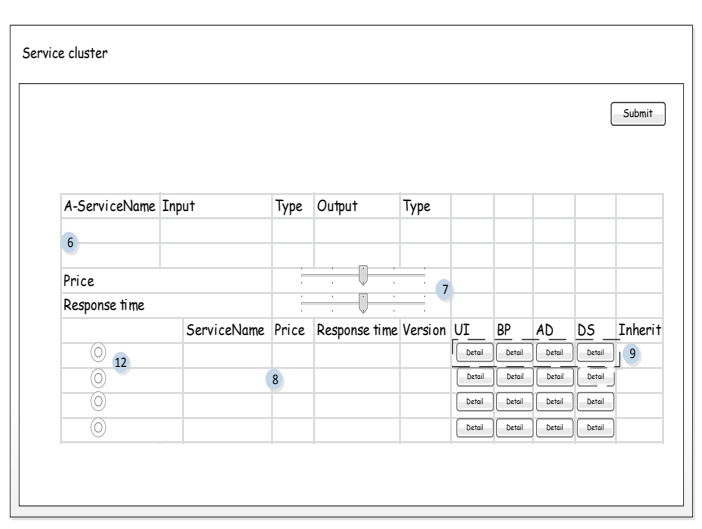

Figure 3. Service Cluster

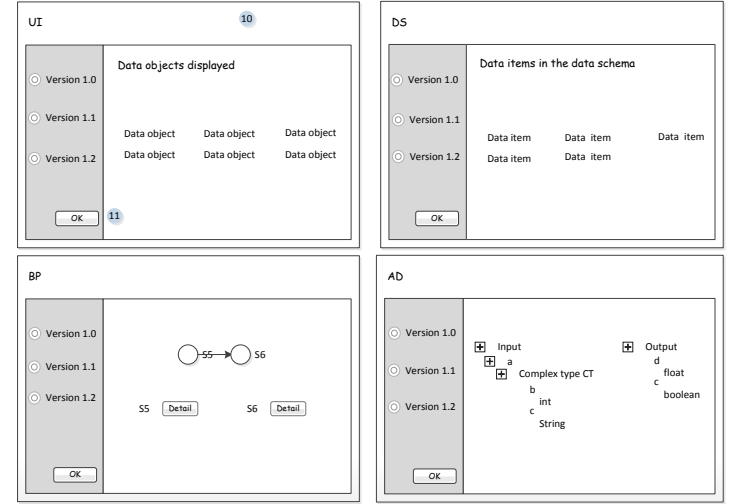

Figure 4. Service Attributes

As shown in Figure 3, each concrete service with a set of attributes can be seen in the Service cluster interface (6). To submit the preferences, users can tune up the bar (7) to 
select services that satisfy QoS requirements (8). As shown in Figure 4, a set of attributes (UI, BP, AD, DS) can be checked (9) (10). Then concrete services can be determined to be mapped after users checking attributes of services of different versions (111 (12).

As shown in Figure 5, when all the concrete services of the abstract services are determined, the concrete services can be displayed when clicking the Map button (13) (14). Then users can customize services they need by modifying attributes (15) (17)). Then the customization information and the services will be updated in the model. The frequent evolution of services to meet business requirements changed always leads to many versions of the same original service stored in the system (ब).

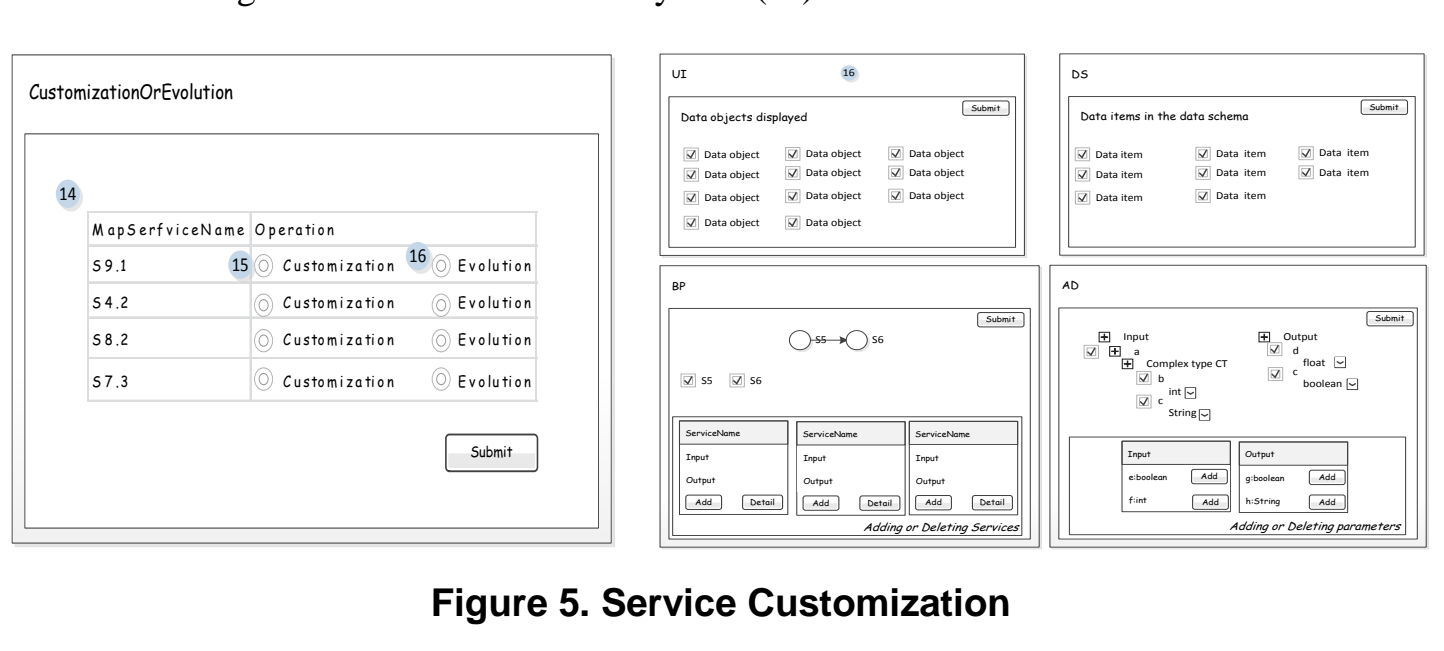

\subsection{Service Granularity Analysis}

To test the effectiveness of granularity on the performance of multi-tenant service composition, we firstly use severaldata sets to do the experiment. For each data set, we have manually created five requirements which can be achieved by services of different granularities ranged from 2 to 10 . We take the average response time of those requirements as the final composition time.

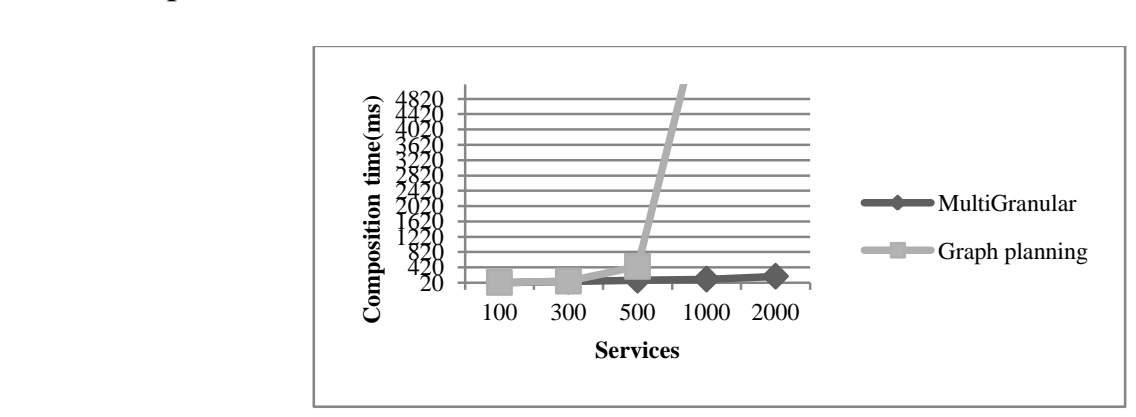

Figure 6. Service Composition Time

Figure 6 shows the results about the composition time for the specified service requirements in services ranged from 100 to 2000. It can be easily concluded that when the number of services is limited to be small, the time taken by the graph planning approach is nearly the same with the MultiGranular-based approach. However, as the number of services grows, graph planning approach takes far more time to find out the composition plans than the MultiGranular-based approach because it involved much semantic-related computing and 
backtracking. On the contrary, the response time trend for MultiGranular-based approach is stable and does not increase much.

Secondly, we use 1000 services and manually created 5 requirements which can be achieved by services of different granularities ranged from10 to 14. Thus, we take the average response time of those requirements as the final composition time. Besides, because of the similarity of semantic concept, users can be provided with many service composition plans. Though those plans can satisfy functionalities needed, the correlation degree will be different. In order to test if our approach can achieve those plans on the basic of graph planning approach as we know it is sure to get suitable ones, we take the correlation degree of service composition plans as a measurement of accuracy. So we test the effectiveness in the view of correlation degree of service composition plans (SCPCD). The formula to compute correlation degree is as follows:

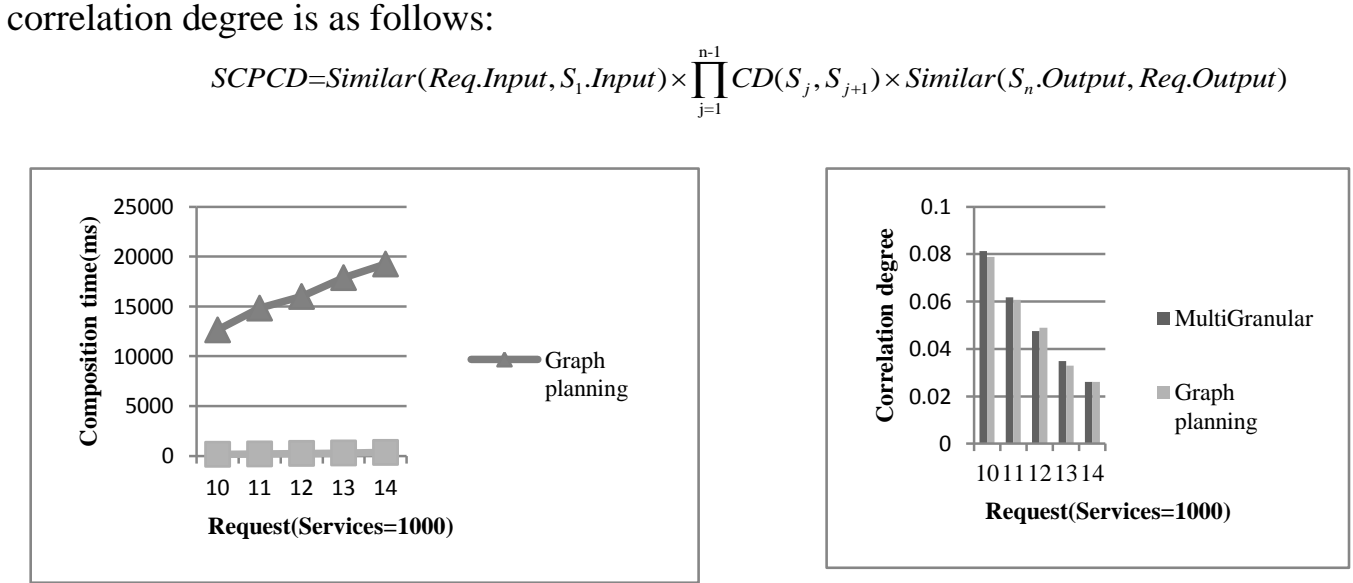

Figure 7.Service Composition Time

Figure 8. Correlation Degree of
Service Composition Plans

Figure 7 shows the results about the composition time for the specified service requirements in services 1000. It can be easily concluded that the response time trend for MultiGranular-based approach is stable and does not increase much while the response time trend for the graph planning approach increases rapidly. As is shown in Figure 8, the MultiGranular-based approach can achieve service composition plans of similar correlation degree with the graph planning approach, which indicates that the MultiGranular-based approach can achieve correct service composition plans but in a short time.

\subsection{Scalability Testing}

The above experiment results of Figure 6, 7 and 8 were conducted under a small service number of services. To illustrate the scalability, we also devised 5 requirements which can be achieved by services of different granularities ranged from 2 to 10 in the service ranged from 3000 to 5000. As we can see in Table 1, when the service number becomes large, it is very hard for graph planning approach to retrieve the composition plans because the searching time blows up exponentially and memories quickly run out. On the contrary, the response time trend for MultiGranular-based approach does not increase much. 


\section{Table 1. Service Composition Time}

\begin{tabular}{|l|l|l|}
\hline & MultiGranular & Graph planning \\
\hline 3000 & $264 \mathrm{~ms}$ & 153340.7 \\
\hline 4000 & $297.6 \mathrm{~ms}$ & -- \\
\hline 5000 & $310.2 \mathrm{~ms}$ & -- \\
\hline
\end{tabular}

\subsection{Analysis of Basic Service Clustering and Correlation Mining}

In order to illustrate the effectiveness of mining correlation relationship in the situation of clustering, we demonstrate the number of mined correlation relationships and the time for that with different service number ranged from 100 to 2000. Figure 9 shows that the time consumed after clustering is much less than that without clustering, and Figure 10 shows that the number of mined correlation relationships is almost the same compared the without clustering. As many services are similar in functionality and are not cørrerated, they can be filtered through clustering for preprocessing.

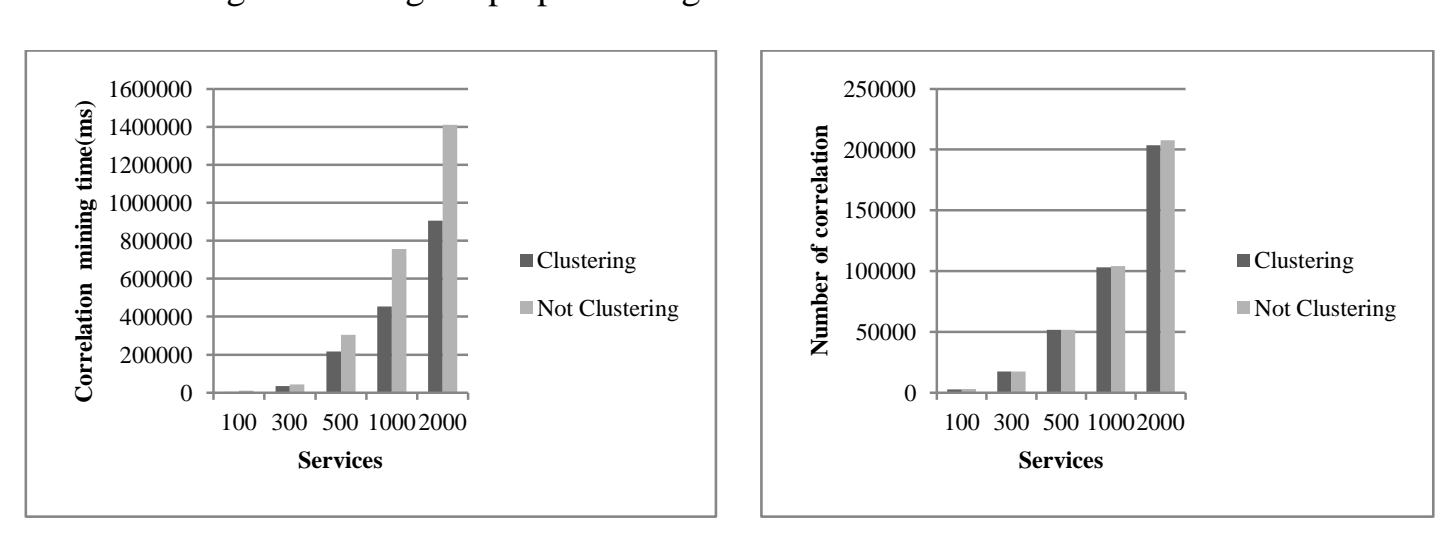

Figure 9. Correlation Mining Jime

\section{Conclusionsand Future Work}

Figure 10. Number of Correlation

Traditional semantic-based composition approaches have not yet well addressed several challenging issues for multi-tenant service composition in cloud computing. In this paper, we have presented an innøvative composition technique by combining clustering and correlation mining together to build MultiGranular as a semantic basis for supporting multi-tenant service composition. Based on MultiGranular, the computational efficiency can be significantly improved yhile guaranteeing the individual and diverse requirement. And frequent service evolution has been also taken into consideration. Besides, our approach can be more scalable to a large scale of service sets and alleviate tenant or developers from the burden of using complicated semantic knowledge for service composition; thus make service composition toward more realistic and easier. An experiment and evaluation has been conducted to demonstrate the effectiveness of our proposed approaches. In future, we will turn our approach to the standardized ontologies on Internet to build more comprehensive MultiGranular. In addition, we will further improve the efficiency of the process of basic services clustering and correlation mining. 


\section{Acknowledgements}

This work has been supported by the National Natural Science Foundation of China under Grant No. 61003253; the Natural Science Foundation of Shandong Province of China under Grant No. ZR2010FM031, ZR2010FQ010.

\section{Reference}

[1] X. Z. Liu and H. Mei, Science China (Information Sciences), vol. 53, no. 1, (2010), pp. 50-63.

[2] S.G. Wang, Q. B. Sun, G. W. Zhang, and F. C. Yang, "Journal of software", vol. 23, no. 6, (2012), pp. 1397-1412.

[3] A. Klein, F. Ishikawa, and S. Honiden, "Towards network-aware service composition in the Cloud", Proceedings of the 21st international conference on World Wide Web, (2012) April 16-20, Lypr, Japan.

[4] K. J. Ren, N. Xiao, and J. J. Chen, "IEEE Transaction on services computing", vol. 4, no. 3, (2011), pp. 216-229.

[5] H. Wada, J. Suzuki, Y. Yamano, and K. Oba, "IEEE Transaction on services Computing", vol. 5, no. 3, (2012), pp. 358-372.

[6] M. Xu, L. Z. Cui, and Q. Z. Li, “Acta Electronica Sinica”, vol 40, no. 7, (2012), pp. 1404-1409.

[7] C. Zeng, X. Guo, W. J. Ou, and D. Han, "Cloud conputing service composition and search based on semantic", Proceedings of the 1st International Conference on Cloud Compating, (2009) December 1-4; Berlin, Germany.

[8] G. B. Zou, Y. X. Chen, Y. Xiang, R. Y. Huang, and Xu, "AI planning and combinatorial optimization for web service composition in cloud computing", Proceedings of the International Conference on Cloud Computing and Virtualization, (2010) May 17 18, Singapore.

[9] B. Zhou, K. Yin, Y. Jiang, H. H., and S. Zhang "Journal of soltware", vol. 6, no. 3, (2011), pp. 366-373.

[10] J. W. Ma, D. K. Guo, J. X. Liu, and X. S. Lyo, "Journal of Harbin Engineering University", vol. 31, no. 10, (2010), pp. 1360-1366.

[11] J. X. Liu, K. Q. He, J. Wang, D. H. Yu, Z.W. Feng, D. Ning, and X. W. Zhang, Chinese Journal of Computers, vol. 36, no. 2, (2013), pp. 238-251.

[12] X. Li, B. Cheng, G.W. Yang, and)Q.H. Liu, Journar of Software”, vol. 20, no. 12, (2009), pp. 3101-3116.

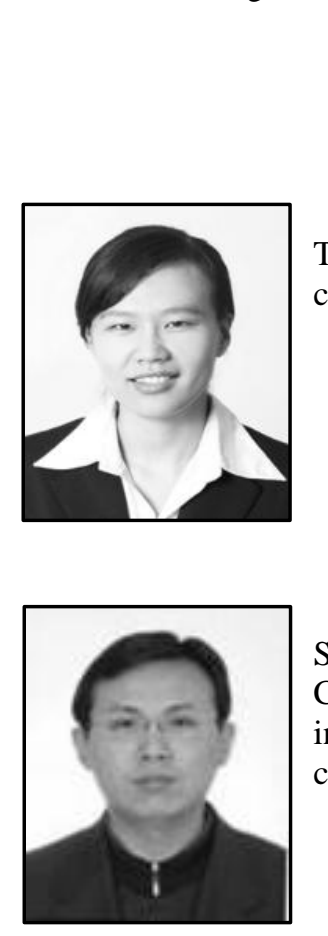

\section{Authors}

Huhu Cai, she is a M.S. candidate in the School of Computer Science and Technology at the Shandong University. Her research interest is service computing.

Lizhen Cui, he received the $\mathrm{PhD}$ degree in computer science from the Shandong University in 2006.He is an associate professor in the School of Computer Science and Technology at Shandong University. His research interests include service computing, workflow and data management for cloud computing 
International Journal of Multimedia and Ubiquitous Engineering Vol.9, No.6 (2014)

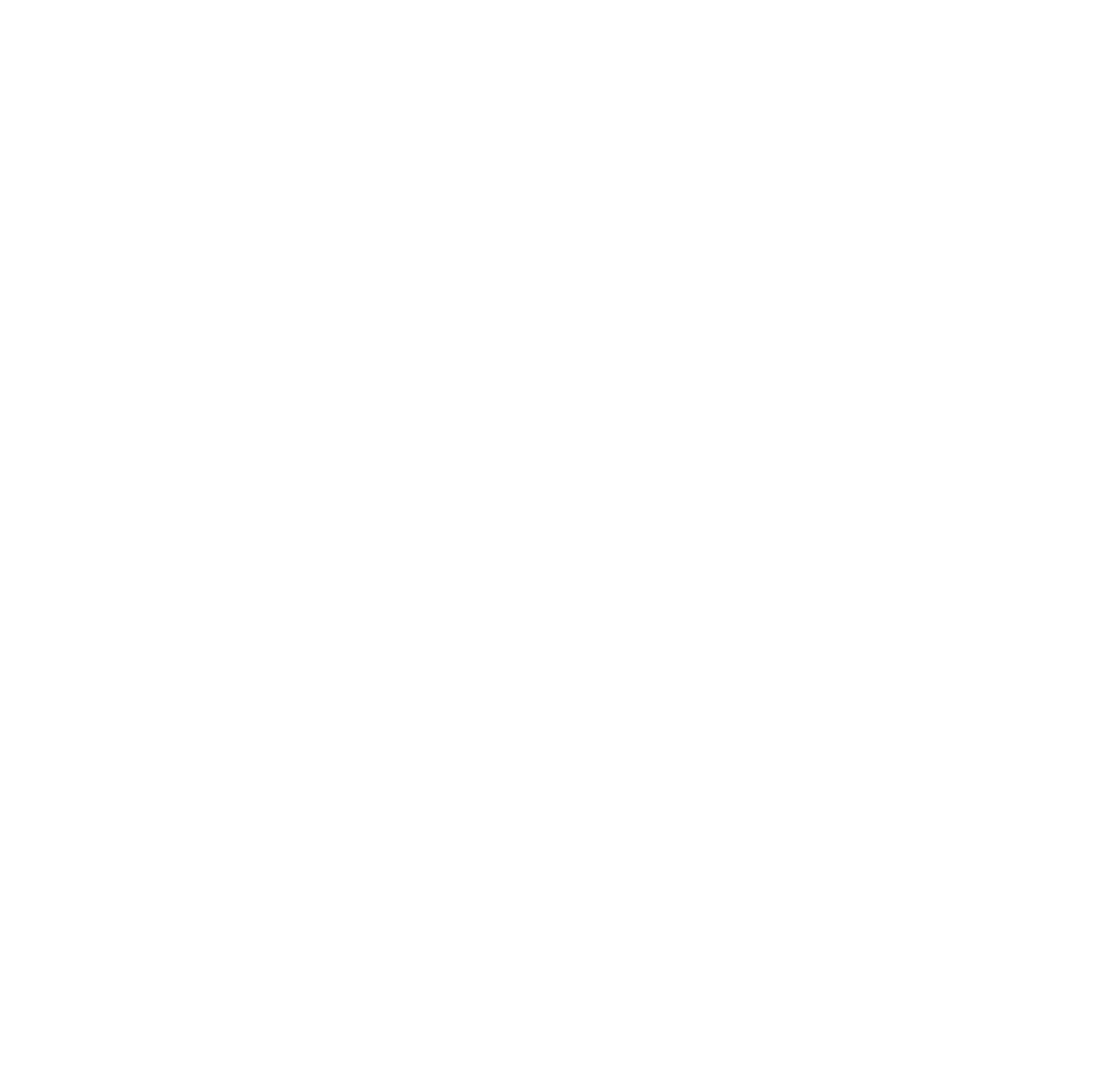

